\title{
Polytypism in Natural SiC Using Laue Microdiffraction at ALS 12.3.2
}

Camelia Veronica $\operatorname{Stan}^{1}$, Earl Francis OBannon ${ }^{2}$, Larissa F. Dobrzhinetskaya ${ }^{3}$, Nobumichi Tamura ${ }^{1}$

${ }^{1}$ Lawrence Berkeley National Lab, Berkeley, United States, ${ }^{2}$ Earth \& Planetary Sciences, University of California Santa Cruz, Santa Cruz, United States, ${ }^{3}$ Department of Earth Sciences, University of California Riverside, Riverside, United States

E-mail: cstan@lbl.gov

Silicon carbide ( $\mathrm{SiC}$, moissanite) is a common industrial material that is rarely found in terrestrial rocks and meteorites. It has been found to adopt over 300 different crystal structures, most of which are polytypic: they consist of alternating layers of Si and $\mathrm{C}$, with only small stacking faults or shears distinguishing them from one another. In nature, only a few polytypes of $\mathrm{SiC}$ have been found, primarily a cubic zincblende type (3C-SiC), several hexagonal wurtzite types (4H-SiC and $6 \mathrm{H}-\mathrm{SiC})$, and a rhombohedral type (15R-SiC). Our natural silicon carbide sample is from a Miocene tuff (Yizre'el Valley, Israel) related to interplate alkaline basalt volcanism. Three SiC grains with native silicon and metal silicide inclusions were analyzed using Raman spectroscopy and synchrotron Laue X-ray microdiffraction accompanied by mapping at a 5-8 um resolution. SiC is found to crystallize in only the $4 \mathrm{H}$ and $6 \mathrm{H}$ polytypes. Due to the crystal orientation of the grains, as well as the significant difference in the c-axis length ( $\sim 10$ vs. $\sim 15 \mathrm{um}$ in $4 \mathrm{H}$ and $6 \mathrm{H}$ respectively), we were able to unambiguously assign polytypes to each diffraction pattern. Each grain contains large areas where one polytype dominates as a single crystal. In some cases, multiple stacking faults and misoriented polycrystalline aggregates of $\mathrm{SiC}$ occur at the $4 \mathrm{H} / 6 \mathrm{H}$ interface. In other cases we see intercalation of the $4 \mathrm{H}$ and $6 \mathrm{H}$ crystals throughout the diffracting volume without a significant change in their crystallographic axes orientation, pointing to a possibly incommensurate crystal structure. Stress and strain are also mapped for all three grains, showing a slight ( $<2 \mathrm{ppt})$ compressive strain in the y direction of all three grains, and a tensile strain in the $x$ and $z$ directions. In the SiC-2 grain, a mostly single-crystalline Si inclusion was found, with an exposed surface diameter of $~ 30$ um. We examine residual strain in Si by both Laue X-ray diffraction and Raman spectroscopy, and find results to generally agree between the two measurements.

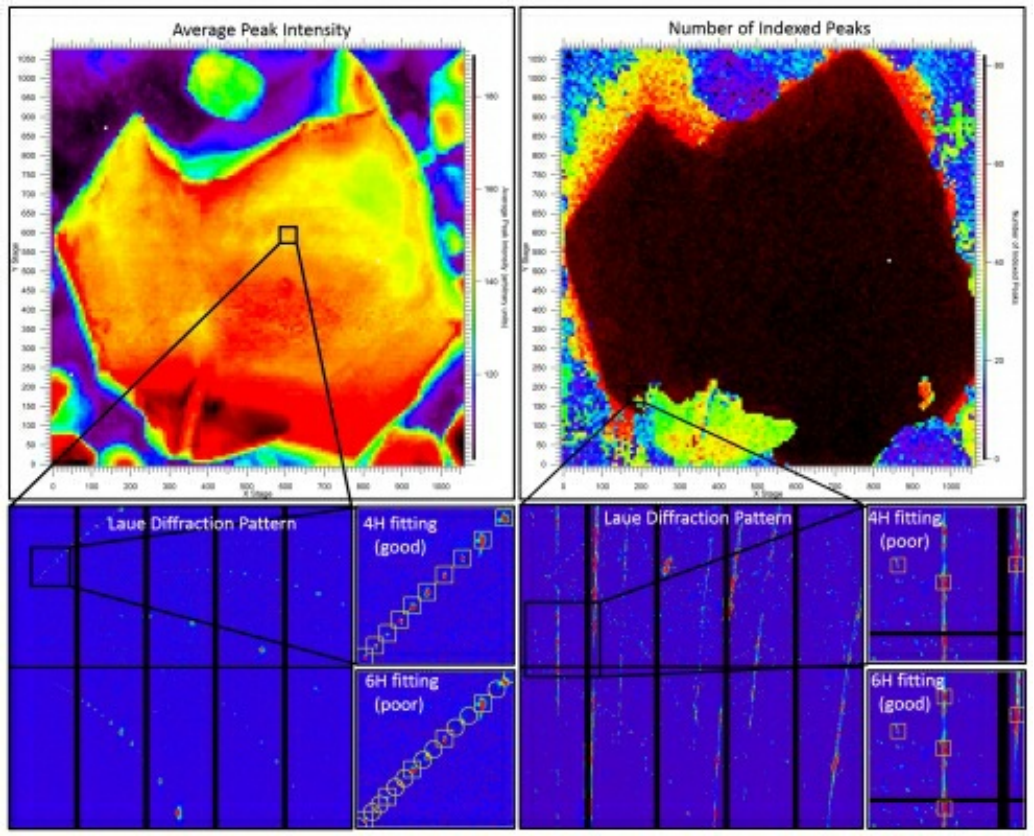

Keywords: Laue Microdiffraction, Silicon Carbide, Synchrotron 